\title{
Amino acid profiles in relation to chronic periodontitis and rheumatoid arthritis
}

\author{
Tetsuo Kobayashi ${ }^{1,2}$, Moe Okada ${ }^{1}$, Satoshi Ito ${ }^{3}$, Daisuke Kobayashi ${ }^{3,4}$, Atsuko Shinhara ${ }^{5}$, \\ Takahiko Muramatsu ${ }^{5}$, Tsuyoshi Kobayashi ${ }^{5}$, Ichiei Narita ${ }^{4}$, Kiyoshi Nakazono ${ }^{3}$, \\ Akira Murasawa $^{3}$, Hiromasa Yoshie ${ }^{1}$
}

${ }^{1}$ Division of Periodontology, Department of Oral Biological Science, Niigata University Graduate School of Medical and Dental Sciences, Niigata, Japan

${ }^{2}$ General Dentistry and Clinical Education Unit, Niigata University Medical and Dental Hospital, Niigata, Japan

${ }^{3}$ Niigata Rheumatic Center, Shibata, Japan

${ }^{4}$ Division of Clinical Nephrology and Rheumatology, Department of Homeostatic Regulation Developments, Niigata University Graduate School of Medical and Dental Sciences, Niigata, Japan

${ }^{5}$ Institute for Innovation, Ajinomoto Co., Inc., Kawasaki, Japan

Email: kotetsuo@dent.niigata-u.ac.jp

Received 6 January 2014; revised 7 February 2014; accepted 16 February 2014

Copyright (C) 2014 Tetsuo Kobayashi et al. This is an open access article distributed under the Creative Commons Attribution License, which permits unrestricted use, distribution, and reproduction in any medium, provided the original work is properly cited. In accordance of the Creative Commons Attribution License all Copyrights ( 2014 are reserved for SCIRP and the owner of the intellectual property Tetsuo Kobayashi et al. All Copyright (C) 2014 are guarded by law and by SCIRP as a guardian.

\section{ABSTRACT}

Chronic periodontitis (CP) and rheumatoid arthritis (RA) are chronic inflammatory conditions, and share many pathologic features. Plasma amino acid profiles have been shown to be associated with RA, but their relevance to $\mathrm{CP}$ remains unclear. The aim of the present study is to evaluate amino acid profiles in relation to CP and RA. The study participants consisted of 62 patients with RA (RA group), 30 patients with CP (CP group) and 29 healthy controls ( $H$ group) in age-, gender-, smoking status-balanced condition. Clinical periodontal and rheumatologic parameter values and plasma levels of 21 amino acids, $C$-reactive protein (CRP), interleukin-6 (IL-6), and tumor necrosis factor-alpha (TNF- $\alpha$ ) were determined. Multiple comparison analyses revealed that the RA group exhibited similar periodontal conditions but significantly higher levels of CRP, IL-6, and TNF- $\alpha$ than the CP group $(P<0.01)$. A total of four amino acids (glycine, histidine, ornithine, and alpha-aminobutyric acid) were significantly different in the concentrations among the three groups $(P<0.01)$. The RA group displayed significantly lower levels of glycine and histidine and significantly higher ornithine level than the $C P$ and $H$ groups $(P<0.01)$. The $C P$ group showed significantly higher alpha-aminobutyric acid level than the RA and $H$ groups $(P<0.01)$. Of these four amino acids, a significantly positive correlation was found between ornithine level and $\%$ of sites with bleeding on probing $(P=0.006)$ in the RA group. These results suggest a possibility that profiles of four amino acids may play a role in the pathogenesis of $\mathrm{CP}$ and RA.

\section{KEYWORDS}

Amino Acid; Chronic Periodontitis; Rheumatoid Arthritis; Case-Control Study

\section{INTRODUCTION}

Similarities in the clinical and pathological features have been suggested between periodontitis and rheumatoid arthritis (RA) [1,2]. Both diseases represent chronic destructive inflammatory diseases characterized by accumulation and persistence of inflammatory infiltrates in the local lesions. Patients with RA were more likely to exhibit periodontits [3,4], while individuals with moderate-to-severe periodontitis had a higher prevalence of RA than those without periodontitis [5,6]. These observations imply that certain features of the inflammatory response are common to both diseases, which might be underpinned by biological pathways [2].

There is evidence to suggest the similarities in the plasma cytokine and gene expression profiles between patients with RA and those with periodontitis $[7,8]$. The pathogenesis of both diseases is also characterized by increased levels of circulating matrix metalloproteinases, reactive oxygen species, lipid mediators, and neutrophil- 
associated enzymes [1,2]. Furthermore, it has been proposed that polymorphisms relating to genes encoding inflammatory cytokines might confer susceptibility to RA and periodontitis [9-11]. These findings suggest an importance to predict individuals at a high risk of developing RA and periodontitis with a circulating biomarker.

Recently, a significance of profiling plasma multiple metabolites including amino acids has been demonstrated for diagnosis of RA [12,13]. It has been reported that patients with RA and psoriatic arthritis could be distinguished with high sensitivity and specificity by detection of amino acids [12]. Additionally, plasma levels of metabolites were shown to be associated with disease activity of RA [13]. However, there is no information to date on amino acid profiles in plasma from patients with periodontitis, although there has been a few earlier studies with analysis of saliva and gingival crevicular fluid from the patients with periodontal disease [14,15]. Therefore, the aim of the present study is to compare plasma levels of amino acids among patients with RA and CP, and healthy individuals.

\section{MATERIAL AND METHOD}

\subsection{Participants}

Sixty-two Japanese adults with RA (53 females and 9 males; mean \pm standard error [SE] age $60.8 \pm 1.6$ years) (RA group) followed at the Niigata Rheumatic Center, Shibata, Japan, were recruited between July 2010 and August 2011 in the present study. All patients were confirmed to fulfill the 1987 revised classification criteria of American Rheumatism Association [16]: 1) morning stiffness in and around joints lasting at least 1 hour before maximal improvement; 2) soft tissue swelling of 3 or more joint areas; 3) swelling of the proximal interphalangeal, metacarpophalangeal, or wrist joints; 4) symmetric swelling; 5) rheumatoid nodules; 6) the presence of rheumatoid factor; and 7) radiographic erosions and/or periarticular osteopenia in hand or wrist joints. Criteria 1) through 4) must have been present for at least 6 weeks. RA was defined by the presence of four and more criteria. All patients fulfilled the following three exclusion criteria: 1) the presence of diabetes mellitus and pregnancy, 2) having antibiotic treatment within the previous 3 months, 3) a history or the presence of any periodontal therapy and mouth rinse usage within the previous 3 months. The duration of RA and medication of corticosteroids, disease-modifying antirheumatic drugs (DMARDs), nonsteroidal anti-inflammatory drugs (NSAIDs), and tumor necrosis factor-alpha (TNF- $\alpha$ ) inhibitors were examined at the time of the examination.

A group of 30 race-, age-, gender-, and smoking status-balanced adults with CP only ( 25 females and 5 male; mean \pm SE age $61.6 \pm 1.4$ years) (CP group) and 29 race-, age-, gender-, and smoking status-balanced healthy individuals with no signs of systemic diseases and periodontitis (24 females and 5 males; mean \pm SE age $54.5 \pm 2.6$ years) (H group) referred to the Periodontics and General Dentistry Clinic of the Niigata University Medical and Dental Hospital, were included July 2010 and August 2011. The presence of periodontitis was defined as having at least one site with clinical attachment level (CAL) $\geq 4 \mathrm{~mm}$ [17]. Individuals who exhibited no signs of periodontitis, as determined by the absence of sites with CAL $>3 \mathrm{~mm}$ were assigned to the $\mathrm{H}$ group. Signed informed consent was obtained from all participants; the format of the study was reviewed and approved of Ethical Committee for the use of Human subjects in research, Niigata University Faculty of Dentistry (No. 22-R9-1006, on June 16, 2010), and Niigata Rheumatic Center (No. 1, on April 26, 2010).

\subsection{Clinical Assessments}

Clinical periodontal assessments were performed by two calibrated examiners (T.K. and M.O.) who were masked from the group assignments and the rheumatologic data. The calibration was performed before the study with 5 volunteer subjects in Niigata University Faculty of Dentistry. Reproducibility of the clinical measurements was calculated by means of the $\kappa$ index, and a value of 0.857 was obtained for CAL with a difference of $\pm 1 \mathrm{~mm}$. All participants were evaluated clinically in the following measurements: number of teeth present, probing depth (PD), CAL, supragingival plaque accumulation, and bleeding on probing (BOP). The presence or absence of supragingival plaque and BOP were recorded at four and six sites around each tooth, respectively. Measurements of PD and CAL were conducted with a Williams probe at six sites around each tooth, recorded to the nearest millimeter, and every observation close to $0.5 \mathrm{~mm}$ was rounded to the lower whole number. The averaged score for whole-mouth PD, CAL, and the number of sites with plaque and BOP divided by the total number of sites per mouth and multiplied by 100 were calculated for each subject.

The disease activity of RA was determined with Disease Activity Score including 28 joints using C-reactive protein (DAS28-CRP), constituting four categories: remission (DAS28-CRP $<2.3)$, low $(2.3 \leq$ DAS28-CRP $<$ $2.7)$, moderate $(2.7 \leq$ DAS28-CRP $<4.1)$, and high disease activity ( $4.1 \leq$ DAS28-CRP), which underestimates disease activity compared with DAS28 using erythrocyte sedimentation rate (DAS28-ESR) in Japan [18]. Smoking status of the participants was classified as currentsmokers, former-smokers, or never-smokers, according to information provided on a standard questionnaire.

\subsection{Measurements of Inflammatory Markers}

Peripheral venous blood samples were obtained from all 
participants and placed on ice within 5 minutes. Plasma was isolated within 4 hours from the blood samples by centrifugation at $1500 \mathrm{~g}$ for 20 minutes, and stored at $-70^{\circ} \mathrm{C}$ until use. Concentrations of rheumatoid factor (RF) and high-sensitive CRP were determined with a latex particle-enhanced and a simple nephelometric method (SRL, Tokyo, Japan). Levels of interleukin-6 (IL-6), TNF- $\alpha$, and anti-cyclic citrullinated peptide (CCP) antibodies were determined by sensitive enzyme-linked immunosorbent assay (ELISA) (IL-6 and TNF- $\alpha$ : R\&D Systems, Minneapolis, MN, USA; anti-CCP: Medical \& Biological Laboratories, Aichi, Japan), according to the manufacturer's instructions. The microtiter plates were read at a wavelength of $450 \mathrm{~nm}$ with an automated microplate reader (Bio-Rad Japan Laboratories, Tokyo, Japan). The lower limits of detection for these measurements were as follows: RF, $1.25 \mathrm{IU} / \mathrm{ml}$; CRP, $0.004 \mathrm{mg} /$ dl; IL-6, $0.016 \mathrm{pg} / \mathrm{ml}$; TNF- $\alpha, 0.038 \mathrm{pg} / \mathrm{ml}$; anti-CCP antibodies, $0.4 \mathrm{U} / \mathrm{ml}$. Positivity of RF and anti-CCP antibodies was defined as showing more than $15 \mathrm{IU} / \mathrm{ml}$ and $4.5 \mathrm{U} / \mathrm{ml}$, respectively. Levels of measurements below the lower limit of detection were recorded as being not determined.

\subsection{Measurements of Amino Acids}

Plasma levels of amino acids were determined with precolumn derivatization high performance liquid chromatography/electrospray mass spectrometry [19]. The following 21 amino acids and related molecules were measured: Glycine (Gly), Alanine (Ala), Serine (Ser), Proline (Pro), Valine (Val), Threonine (Thr), Isoleucine (Ile), Leucine (Leu), Aspragine (Asn), Glutamine (Gln), Glutamic Acid (Glu), Methionine (Met), Histidine (His), Phenylalanine (Phe), Arginine (Arg), Citrulline (Cit), Tyrosine (Tyr), Tryptophan (Trp), Ornithine (Orn), Lysine (Lys), alpha-aminobutyric acid ( $\alpha$-ABA).

\subsection{Statistical Analyses}

The required sample size was more than 15 subjects in each group, which was based on power calculation test with repeated-measures analysis of variance with $5 \%$ of alpha level, 0.14 of obtained effect size, 0.8 of statistical power level. After evaluating the normality of distribution by Kolmogorov-Smirnov test, differences in clinical and laboratory parameter values were assessed among the three groups by Kruskal-Wallis test, and when significant differences were detected, further assessment of differences between the two groups were done by Scheffe's test. The Spearman's rank correlation coefficient analysis was used to determine the relationship between levels of amino acids and periodontal conditions. Statistical significance was accepted at $1 \%(p<0.01)$.

\section{RESULTS}

Table 1 shows the demographic, clinical, and laboratory parameter values of RA, CP, and H groups. Both the RA and CP groups exhibited significantly less number of teeth and higher scores of periodontal parameters (\% of sites with plaque and BOP, PD, CAL, and \% sites with $\mathrm{PD}$ and $\mathrm{CAL} \geq 4 \mathrm{~mm})$ than the $\mathrm{H}$ group $(P<0.01)$. However, these dental parameter values proved comparable between the RA and CP groups $(P>0.01)$. Levels of CRP, IL-6, and TNF- $\alpha$ were significantly higher in the RA group than those in the $\mathrm{CP}$ and $\mathrm{H}$ groups $(P<0.01)$.

As shown Table 2, levels of four amino acids (Gly, His, Orn, and $\alpha$-ABA) were significantly different among the three groups $(P<0.01)$. The RA group displayed significantly lower levels of Gly and His and significantly higher Orn level than the $\mathrm{CP}$ and $\mathrm{H}$ groups ( $P$ $<0.01)$. On the other hand, the CP group showed significantly higher $\alpha$-ABA level than the RA and $\mathrm{H}$ groups ( $P$ $<0.01$ ).

Of these four amino acids, a significantly positive correlation was found between Orn level and \% of sites with BOP $(P=0.006)$ in the RA group (Table 3$)$. No associations were obtained between amino acid levels and periodontal conditions in the CP group $(P>0.01)$ (Table 3).

\section{DISCUSSION}

The percentage of females was $85.5 \%$, the mean age was 60.8 years, and the mean RA duration was 11.6 years in the RA group in the present study. These observations are almost in accordance with the results of race-matched large-cohort (7512 patients) IORRA and (3073 patients) Ninja studies [20,21]. The frequencies of patients with remission, low-, moderate-, and high-activity, as measured with DAS28-CRP, as well as RA medication were almost comparable between the present and other studies [20,21]. Furthermore, patients with RA exhibited higher levels of CRP, IL- 6 , and TNF- $\alpha$ than the age-, gender-, smoking status-, and periodontal condition-balanced individuals with $\mathrm{CP}$, which is consistent with the results of previous study [8]. These findings imply that patients with RA who participated in the current study were representative of the general Japanese RA population.

Determination of the plasma concentrations of amino acids is important for understanding the pathophysiology of inflammatory diseases such as RA and periodontitis. However, it is necessary to pay an attention for management of blood samples. It has been demonstrated that delays in processing of blood samples caused a rapid change in amino acid concentrations [22]. Therefore, in the present study, blood samples were placed on ice within 5 minutes after collection, and plasma was isolated within 4 hours to avoid degradation of amino acids. 
Table 1. Demographic, clinical, and laboratory characteristics of patients with rheumatoid arthritis (RA) and chronic periodontitis (CP), and healthy controls (H).

\begin{tabular}{|c|c|c|c|}
\hline Parameters & $\begin{array}{l}\text { RA group } \\
(\mathrm{n}=62)\end{array}$ & $\begin{array}{l}\text { CP group } \\
(\mathrm{n}=30)\end{array}$ & $\begin{array}{l}\text { H group } \\
(\mathrm{n}=29)\end{array}$ \\
\hline \multicolumn{4}{|l|}{ Demographic } \\
\hline Age (years; mean \pm SE) & $60.8 \pm 1.6$ & $61.6 \pm 1.4$ & $54.5 \pm 2.6$ \\
\hline Female (n [\%]) & $53(85.5)$ & $25(83.3)$ & $24(82.8)$ \\
\hline Smoker of current/former/never (\%) & $0 / 21 / 79$ & $0 / 20 / 80$ & $0 / 0 / 100$ \\
\hline \multicolumn{4}{|l|}{ Clinical (dental) } \\
\hline Number of teeth present (mean \pm SE) & $22.6 \pm 0.6^{*}$ & $22.6 \pm 1.0^{*}$ & $28.3 \pm 0.3$ \\
\hline$\%$ sites with plaque (mean $\pm \mathrm{SE}$ ) & $31.2 \pm 1.7^{*}$ & $34.1 \pm 3.5^{*}$ & $8.1 \pm 1.2$ \\
\hline$\%$ sites with BOP (mean \pm SE) & $22.3 \pm 3.1^{*}$ & $15.7 \pm 3.0^{*}$ & $2.0 \pm 0.4$ \\
\hline $\mathrm{PD}(\mathrm{mm} ;$ mean $\pm \mathrm{SE})$ & $2.9 \pm 0.1^{*}$ & $2.6 \pm 0.1^{*}$ & $2.2 \pm 0.1$ \\
\hline$\%$ sites with $\mathrm{PD} \geq 4 \mathrm{~mm}$ (mean $\pm \mathrm{SE}$ ) & $19.0 \pm 2.4^{*}$ & $13.2 \pm 2.1^{*}$ & $0.0 \pm 0.0$ \\
\hline CAL $(\mathrm{mm}$; mean $\pm \mathrm{SE})$ & $3.0 \pm 0.1^{*}$ & $2.9 \pm 0.1^{*}$ & $2.2 \pm 0.1$ \\
\hline$\%$ sites with $\mathrm{CAL} \geq 4 \mathrm{~mm}($ mean $\pm \mathrm{SE})$ & $24.1 \pm 2.8^{*}$ & $16.7 \pm 2.5^{*}$ & $0.0 \pm 0.0$ \\
\hline \multicolumn{4}{|l|}{ Clinical (rheumatologic) } \\
\hline Duration of RA (years; mean \pm SE) & $11.6 \pm 1.6$ & NA & NA \\
\hline DAS28-CRP (mean \pm SE) & $2.34 \pm 0.10$ & NA & NA \\
\hline \multicolumn{4}{|l|}{ DAS28-CRP category } \\
\hline Remission/Low-/Moderate-/High-activity (\%) & $52 / 21 / 22 / 5$ & NA & NA \\
\hline \multicolumn{4}{|l|}{ Medication } \\
\hline Corticosteroids (n [\%]) & $32(51.6)^{*}$ & $0(0.0)$ & $0(0.0)$ \\
\hline DMARDs (n [\%]) & $42(67.7)^{*}$ & $0(0.0)$ & $0(0.0)$ \\
\hline NSAIDs (n [\%]) & $11(17.7)^{*}$ & $0(0.0)$ & $0(0.0)$ \\
\hline TNF-inhibitors (n [\%]) & $10(16.1)^{*}$ & $0(0.0)$ & $0(0.0)$ \\
\hline \multicolumn{4}{|l|}{ Laboratory } \\
\hline RF levels (IU/ml; mean \pm SE) & $68.1 \pm 10.7$ & NA & NA \\
\hline RF positive (n [\%]) & $43(69.4)$ & NA & NA \\
\hline Anti-CCP titer (U/mL; mean \pm SE) & $146.4 \pm 16.4$ & NA & NA \\
\hline Anti-CCP antibody positive (n [\%]) & $50(80.6)$ & NA & NA \\
\hline CRP levels (mg/dL; mean \pm SE) & $0.41 \pm 0.07^{* * *}$ & $0.18 \pm 0.03$ & $0.04 \pm 0.01$ \\
\hline IL-6 levels $(\mathrm{pg} / \mathrm{ml}$; mean $\pm \mathrm{SE})$ & $4.51 \pm 0.56^{* *}$ & $1.67 \pm 0.17$ & $0.38 \pm 0.05$ \\
\hline TNF- $\alpha$ levels (pg/ml; mean \pm SE) & $8.70 \pm 1.69^{* *}$ & $2.12 \pm 0.33$ & $1.16 \pm 0.07$ \\
\hline
\end{tabular}

BOP = bleeding on probing; PD = probing depth; CAL = Clinical attachment level; DAS28-CRP = disease activity score including 28 joints using C-reactive protein; DMARDs = disease-modifying antirheumatic drugs; NSAIDs = non-steroidal anti-inflammatory drugs; TNF = tumor necrosis factor; RF = rheumatoid factor; Anti-CCP = anti-cyclic citrullinated peptide; IL-6 = interleukin-6; NA = not applicable; *Significantly different from the H group (Kruskal-Wallis and Scheffe's tests, $P<0.01$ ); ${ }^{* *}$ Significantly different from the CP and H groups (Kruskal-Wallis and Scheffe's tests, $P<0.01$ ).

The present results indicated decreased levels of Gly and His in patients with RA compared with individuals with CP and healthy controls, which is supported by the results of other studies [12,23,24]. It has been docu- 
Table 2. Amino acid levels in patients with rheumatoid arthritis (RA) and chronic periodontitis (CP), and healthy controls (H).

\begin{tabular}{|c|c|c|c|}
\hline Amino acid & $\begin{array}{l}\text { RA group } \\
(\mathrm{n}=62)\end{array}$ & $\begin{array}{l}\text { CP group } \\
(\mathrm{n}=30)\end{array}$ & $\begin{array}{l}\text { H group } \\
(\mathrm{n}=29)\end{array}$ \\
\hline \multicolumn{4}{|l|}{ Non-essential amino acid } \\
\hline Glycine (Gly) & $237.0 \pm 8.1^{*}$ & $277.2 \pm 20.3$ & $264.3 \pm 14.3$ \\
\hline Alanine (Ala) & $383.8 \pm 13.9$ & $424.1 \pm 17.4$ & $403.7 \pm 16.1$ \\
\hline Serine (Ser) & $110.9 \pm 3.7$ & $115.4 \pm 6.0$ & $121.3 \pm 4.2$ \\
\hline Proline (Pro) & $167.6 \pm 6.9$ & $171.5 \pm 10.2$ & $183.9 \pm 13.0$ \\
\hline Aspragine (Asn) & $50.4 \pm 1.6$ & $54.8 \pm 2.6$ & $52.6 \pm 2.3$ \\
\hline Glutamine (Gln) & $560.4 \pm 9.1$ & $617.0 \pm 12.4$ & $600.0 \pm 14.8$ \\
\hline Glutamic Acid (Glu) & $36.7 \pm 2.3$ & $31.7 \pm 2.8$ & $27.8 \pm 1.7$ \\
\hline Arginine (Arg) & $92.8 \pm 3.0$ & $104.8 \pm 4.9$ & $95.2 \pm 4.9$ \\
\hline Tyrosine (Tyr) & $69.5 \pm 2.1$ & $74.4 \pm 3.2$ & $69.0 \pm 3.7$ \\
\hline \multicolumn{4}{|l|}{ Essential amino acid } \\
\hline Valine (Val) & $219.9 \pm 6.0$ & $239.6 \pm 7.7$ & $230.3 \pm 8.4$ \\
\hline Threonine (Thr) & $126.2 \pm 4.4$ & $135.3 \pm 7.6$ & $128.0 \pm 5.4$ \\
\hline Isoleucine (Ile) & $63.3 \pm 2.3$ & $72.1 \pm 3.2$ & $70.0 \pm 3.5$ \\
\hline Leucine (Leu) & $110.1 \pm 3.8$ & $127.0 \pm 4.9$ & $122.1 \pm 4.8$ \\
\hline Methionine (Met) & $22.0 \pm 0.9$ & $27.0 \pm 1.5$ & $24.0 \pm 1.2$ \\
\hline Histidine (His) & $72.5 \pm 1.6^{*}$ & $81.7 \pm 2.2$ & $85.4 \pm 2.2$ \\
\hline Phenylalanine (Phe) & $74.8 \pm 2.6$ & $67.0 \pm 2.3$ & $65.9 \pm 2.4$ \\
\hline Tryptophan (Trp) & $53.2 \pm 1.6$ & $55.2 \pm 1.9$ & $59.5 \pm 1.8$ \\
\hline Lysine (Lys) & $178.3 \pm 5.7$ & $199.1 \pm 6.5$ & $182.1 \pm 6.6$ \\
\hline \multicolumn{4}{|l|}{ Other } \\
\hline Citrulline (Cit) & $32.1 \pm 1.6$ & $35.1 \pm 2.0$ & $30.5 \pm 2.2$ \\
\hline Ornithine (Orn) & $74.4 \pm 2.4^{*}$ & $62.4 \pm 2.5$ & $58.8 \pm 3.0$ \\
\hline $\begin{array}{l}\text { alpha-aminobutyric acid } \\
(\alpha-\mathrm{ABA})\end{array}$ & $13.0 \pm 0.5$ & $18.0 \pm 1.1^{* *}$ & $14.1 \pm 0.9$ \\
\hline $\begin{array}{l}\text { Sum of non-essential } \\
\text { amino acid }\end{array}$ & $190.1 \pm 7.4$ & $209.4 \pm 11.9$ & $202.4 \pm 11.6$ \\
\hline $\begin{array}{l}\text { Sum of essential amino } \\
\text { acid }\end{array}$ & $102.4 \pm 2.8$ & $112.0 \pm 4.4$ & $109.1 \pm 4.2$ \\
\hline
\end{tabular}

Values represent the mean $\pm \mathrm{SE}(\mu \mathrm{mol} / \mathrm{L})$; ${ }^{*}$ Significantly different from the $\mathrm{CP}$ and $\mathrm{H}$ groups (Kruskal-Wallis and Scheffe's tests, $P<0.01$ ); ${ }^{* *}$ Significantly different from the RA and $\mathrm{H}$ groups (Kruskal-Wallis and Scheffe's tests, $P<0.01$ ).

mented that patients with RA exhibited lower levels of His than healthy individuals [12]. The decreased His level in RA has been partially explained by increased degradation of His through the His decarboxylase [23]. It has also been reported that the decrease in plasma Gly levels was observed in patients with RA, whose significance in the pathogenesis of RA has not been discussed
Table 3. Correlations between amino acid levels and periodontal conditions in patients with rheumatoid arthritis (RA) and chronic periodontitis (CP).

\begin{tabular}{lccc}
\hline & \% sites with BOP & PD & CAL \\
\hline RA group (n = 62) & & & \\
Glycine (Gly) & $0.51(-0.08)$ & $0.18(-0.17)$ & $0.53(-0.08)$ \\
Histidine (His) & $0.77(+0.04)$ & $0.99(+0.003)$ & $0.75(-0.04)$ \\
rnithine (Orn) & $\mathbf{0 . 0 0 6 ( + 0 . 3 5 )})^{*}$ & $0.31(+0.13)$ & $0.41(+0.11)$ \\
$\begin{array}{l}\text { alpha-aminobutyric } \\
\text { acid }(\alpha \text {-ABA) }\end{array}$ & $0.34(-0.12)$ & $0.31(+0.13)$ & $0.41(-0.11)$ \\
CP group (n=30) & & & \\
Glycine (Gly) & $0.16(-0.26)$ & $0.78(-0.05)$ & $0.43(-0.14)$ \\
Histidine (His) & $0.19(-0.24)$ & $0.31(-0.19)$ & $0.13(-0.28)$ \\
$\begin{array}{l}\text { Ornithine (Orn) } \\
\text { alpha-aminobutyric } \\
\text { acid }(\alpha \text {-ABA) }\end{array}$ & $0.58(+0.10)$ & $0.68(-0.08)$ & $0.45(-0.14)$ \\
\hline
\end{tabular}

$\mathrm{BOP}=$ bleeding on probing; $\mathrm{PD}=$ probing depth; $\mathrm{CAL}=$ Clinical attachment level; Data represent the $p$-value, and correlation coefficient in the parenthesis; "Significantly correlated between the amino acid concentrations and periodontal conditions, as assessed by Spearman's rank correlation analysis $(P<0.01)$.

[24].

Notably, the present data showed that Orn level was increased in patients with RA compared with healthy individuals, which is different from the results of other study [24]. Additionally, Orn level was positively correlated with periodontal inflammation as determined with BOP in patients with RA, but not in individuals with CP and healthy controls. To best of our knowledge, this is the first study demonstrating an association of Orn profile with CP and RA. However, it cannot be concluded from these findings alone whether Orn plays a role in the pathogenesis of RA and periodontitis. Further studies would be required to evaluate longitudinally the effects of Orn and its metabolism on periodontal condition in patients with RA.

Interestingly, similar amino acid profiles were obtained in the present study between individuals with $\mathrm{CP}$ and healthy controls, except for $\alpha$-ABA. These observations are different from earlier studies showing that patients with periodontal disease exhibited considerable amino acid variations in saliva and gingival crevicular fluid $[14,15]$. These inconsistent results might be partly explained by clinical periodontal condition or blood sample management between the present and other studies $[14,15]$. It has been reported that $\alpha$-ABA did not take part in protein synthesis and was only associated with sepsis [25]. Therefore, it would be necessary to perform the clinical and in vitro studies to confirm and extend the observations obtained in the present study. 


\section{CONCLUSION}

The results of the present study suggest a possibility that profiles of four amino acids (Gly, His, Orn, and $\alpha$-ABA) may play a role in the pathogenesis of $\mathrm{CP}$ and RA.

\section{ACKNOWLEDGEMENTS}

The authors declare no conflict of interest related to this study. This work was supported by Grant-in Aid for Scientific Research A (No. 25253104) and C (No. 22592309) and Grant-in Aid for Challenging Exploratory Research (No. 24659922) from the Japan Society for the Promotion of Science, Tokyo, Japan.

\section{REFERENCES}

[1] Mercado, F.B., Marshall, R.I. and Bartold, P.M. (2003) Inter-relationship between rheumatoid arthritis and periodontal disease. A review. Journal of Clinical Periodontology, 30, 761-772.

http://dx.doi.org/10.1034/j.1600-051X.2003.00371.x

[2] de Pablo, P., Chapple, I.L.C., Buckley, C.D. and Dietrich, T. (2009) Periodontitis in systemic rheumatic diseases. Nature Reviews Rheumatology, 5, 218-224. http://dx.doi.org/10.1038/nrrheum.2009.28

[3] Pischon, N., Pischon, T., Kröger, J., et al. (2008) Association among rheumatoid arthritis, oral hygiene, and periodontitis. Journal of Periodontology, 79, 979-986. http://dx.doi.org/10.1902/jop.2008.070501

[4] Dissick, A., Redman, R.S., Jones, M., et al. (2010) Association of periodontitis with rheumatoid arthritis: A pilot study. Journal of Periodontology, 81, 223-230. http://dx.doi.org/10.1902/jop.2009.090309

[5] Mercado, F., Marshall, R.I., Klestov, A.C. and Bartold, P.M. (2000) Is there a relationship between rheumatoid arthritis and periodontal disease? Journal of Clinical Periodontology, 27, 267-272. http://dx.doi.org/10.1034/j.1600-051x.2000.027004267.x

[6] Demmer, R.T., Molitor, J.A., Jacobs Jr., D.R. and Michalowicz, B.S. (2011) Periodontal disease, tooth loss and incident rheumatoid arthritis: Results from the First National Health and Nutritional Examination Survey and its epidemiological follow-up study. Journal of Clinical Periodontology, 38, 998-1006. http://dx.doi.org/10.1111/j.1600-051X.2011.01776.x

[7] Sørensen, L.K., Havemose-Poulsen, A., Bendtzen, K. and Holmstrup, P. (2009) Aggressive periodontitis and chronic arthritis: Blood mononuclear cell gene expression and plasma protein levels of cytokines and cytokine inhibitors. Journal of Periodontology, 80, 282-289. http://dx.doi.org/10.1902/jop.2009.080347

[8] Kobayashi, T., Murasawa, A., Komatsu, Y., et al. (2010) Serum cytokine and periodontal profiles in relation to disease activity of rheumatoud arthritis in Japanese adults. Journal of Periodontology, 80, 650-657. http://dx.doi.org/10.1902/jop.2010.090688

[9] Havemose-Poulsen, A., Sørensen, L.K., Bendtzen, K. and Holmstrup, P. (2007) Polymorphisms within the IL-1 gene cluster: Effects on cytokine profiles in peripheral blood and whole blood cell cultures of patients with aggressive periodontitis, juvenile idiopathic arthritis, and rheumatoid arthritis. Journal of Periodontology, 78, 475492. http://dx.doi.org/10.1902/jop.2007.060135

[10] Kobayashi, T., Ito, S., Kuroda, T., et al. (2007) The interleukin-1 and Fc $\gamma$ receptor gene polymorphisms in Japanese patients with rheumatoid arthritis and periodontitis. Journal of Periodontology, 78, 2311-2318.

http://dx.doi.org/10.1902/jop.2007.070136

[11] Kobayashi, T., Murasawa, A., Ito, S., et al. (2009) Cytokine gene polymorphisms associated with rheumatoid arthritis and periodontitis in Japanese adults. Journal of Periodontology, 80, 792-799. http://dx.doi.org/10.1902/jop.2009.080573

[12] Madsen, R.K., Lundstedt, T., Gabrielsson, J., et al. (2011) Diagnostic properties of metabolic perturbations in rheumatoid arthritis. Arthritis Research \& Therapy, 13, R19. http://dx.doi.org/10.1186/ar3243

[13] Madsen, R.K., Rantapää-Dahlqvist, S., Lundstedt, T., Moritz, T. and Trygg, J. (2012) Metabolic response to change in disease activity during tumor necrosis factor inhibition in patients with rheumatoid arthritis. Journal of Proteome Research, 11, 3796-3804. http://dx.doi.org/10.1021/pr300296v

[14] Syrjänen, S., Piironen, P. and Markkanen, H. (1987) Free amino-acid content of wax-stimulated human whole saliva as related to periodontal disease. Archives of Oral Biology, 32, 607-610. http://dx.doi.org/10.1016/0003-9969(87)90032-X

[15] Syrjänen, S., Alakuijala, L., Alakuijala, P., Markkanen, S.O. and Markkanen, H. (1990) Free amino-acid levels in oral fluids of normal subjects and patients with periodontal disease. Archives of Oral Biology, 35, 189-193. http://dx.doi.org/10.1016/0003-9969(90)90054-E

[16] Arnett, F.C., Edworthy, S.M., Bloch, D.A., et al. (1988) The American rheumatism association 1987 revised criteria for the classification of rheumatoid arthritis. Arthritis \& Rheumatology, 31, 315-324. http://dx.doi.org/10.1002/art.1780310302

[17] Esen, Ç., Alkan, B.A., Kırnap, M., Akgül, Ö., Işıkoğlu, S. and Erel, Ö. (2012) The effects of chronic periodontitis and rheumatoid arthritis on serum and gingival crevicular fluid total antioxidant/oxidant status and oxidative stress index. Journal of Periodontology, 83, 773-779. http://dx.doi.org/10.1902/jop.2011.110420

[18] Inoue, E., Yamanaka, H., Hara, M., Tomatsu, T. and Kamatani, N. (2007) Comparison of disease activity score (DAS) 28-erythrocyte sedimentation rate and DAS28-Creactive protein threshold values. Annals of the Rheumatic Diseases, 66, 407-409. http://dx.doi.org/10.1136/ard.2006.054205

[19] Shimbo, K., Kubo, S., Harada, Y., et al. (2010) Automated precolumn derivatization system for analyzing physiological amino acids by liquid chromatography/ mass spectrometry. Biomedical Chromatography, 24, 683691. http://dx.doi.org/10.1002/bmc.1346

[20] Yamanaka, H., Inoue, E., Singh, G., et al. (2007) Improvement of disease activity of rheumatoid arthritis pa- 
tients from 2000 to 2006 in a large observational cohort study IORRA in Japan. Modern Rheumatology, 17, 283289. http://dx.doi.org/10.1007/s10165-007-0587-6

[21] Matsui, T., Kuga, Y., Kaneko, A., et al. (2007) Disease activity score 28 (DAS28) using C-reactive protein underestimates disease activity and overestimates EULAR response criteria compared with DAS28 using erythrocyte sedimentation rate in a large observational cohort of rheumatoid arthritis patients in Japan. Annals of the Rheumatic Diseases, 66, 1221-1226. http://dx.doi.org/10.1136/ard.2006.063834

[22] Davis, J.S., Darcy, C.J., Piera, K., McNeil, Y.R., Woodberry, T. and Anstey, N.M. (2009) Ex-vivo changes in amino acid concentrations from blood stored at room temperature or on ice: Implications for arginine and taurine measurements. BMC Clinical Pathology, 9, 10. http://dx.doi.org/10.1186/1472-6890-9-10

[23] Sittons, N.G., Dixon, J.S., Astbury, C., Francis, R.J., Bird, H.A. and Wright, V. (1988) Kinetic investigations into the possible cause of low serum histidine in rheumatoid arthritis. Annals of the Rheumatic Diseases, 47, 48-52. http://dx.doi.org/10.1136/ard.47.1.48

[24] Jones, M.G., Cooper, E., Amjad, S., Goodwin, C.S., Barron, J.L. and Chalmers, R.A. (2005) Urinary and plasma organic acids and amino acids in chronic fatigue syndrome. Clinica Chimica Acta, 361, 150-158. http://dx.doi.org/10.1016/j.cccn.2005.05.023

[25] Chiarla, C., Giovannini, I. and Siegel, J.H. (2011) Characterization of alpha-amino-n-butyric acid correlations in sepsis. Translational Research, 158, 328-333. http://dx.doi.org/10.1016/j.trsl.2011.06.005 